\title{
A Closed Inhalation System for Pharmacokinetic and Metabolism Studies of Volatile Compounds with Small Laboratory Animals
}

\author{
W.K. Lutz and Ch. Schlatter \\ Institut für Toxikologie, Eidgenössische Technische Hochschule und Universität Zürich, \\ CH-8603 Schwerzenbach, Switzerland
}

\begin{abstract}
In the inhalation system described an animal can be kept in the same atmosphere of a 2-liter desiccator for up to $24 \mathrm{~h}$. The expired carbon dioxide is adsorbed with soda lime and the resulting reduced pressure is balanced by a supply of oxygen also used for the inflow of the chemical to be investigated. Urine and faeces can be collected separately and the system allows a periodical control of the concentration of the chemical by sampling the air with needle and syringe.
\end{abstract}

The exposure systems used for inhalation toxicity studies are usually dynamic, i.e., the animals are placed in a chamber through which passes a stream of air containing the substance to be investigated (Gage, 1970). This type of system is appropriate for the assessment of the toxic effects of a chemical on the animal. For pharmacokinetic studies on the fate of the chemical in the organism, this type of system is much less useful, since it is not possible to measure the retention of a compound in the body and to determine the rate of metabolism directly. Secondly', for metabolic studies of volatile radioactively labeled compounds, the uptake of the substance should be carried to completion since none of the precious chemical can be afforded to be lost by expiration.

For such cases it would be advantageous to expose the animals to a static atmosphere in an enclosed chamber into which a known amount of the material is dispersed and where the only gas exchange is the replacement of the expired carbon dioxide by oxygen. Since there is no detailed description available for this type of chamber we report our experience with a system set together in our laboratory and shown schematically in Figure 1.

The chamber (A) is a regular desiccator. The animal is held on the standard punched porcelain disk with the holes small enough for retaining the faeces. Urine runs down the walls of the chamber and can at the end of the exposure be collected from the bottom. On the bottom is a crystallizing dish (B) which contains the soda lime necessary for the adsorption of the expired carbon dioxide. 


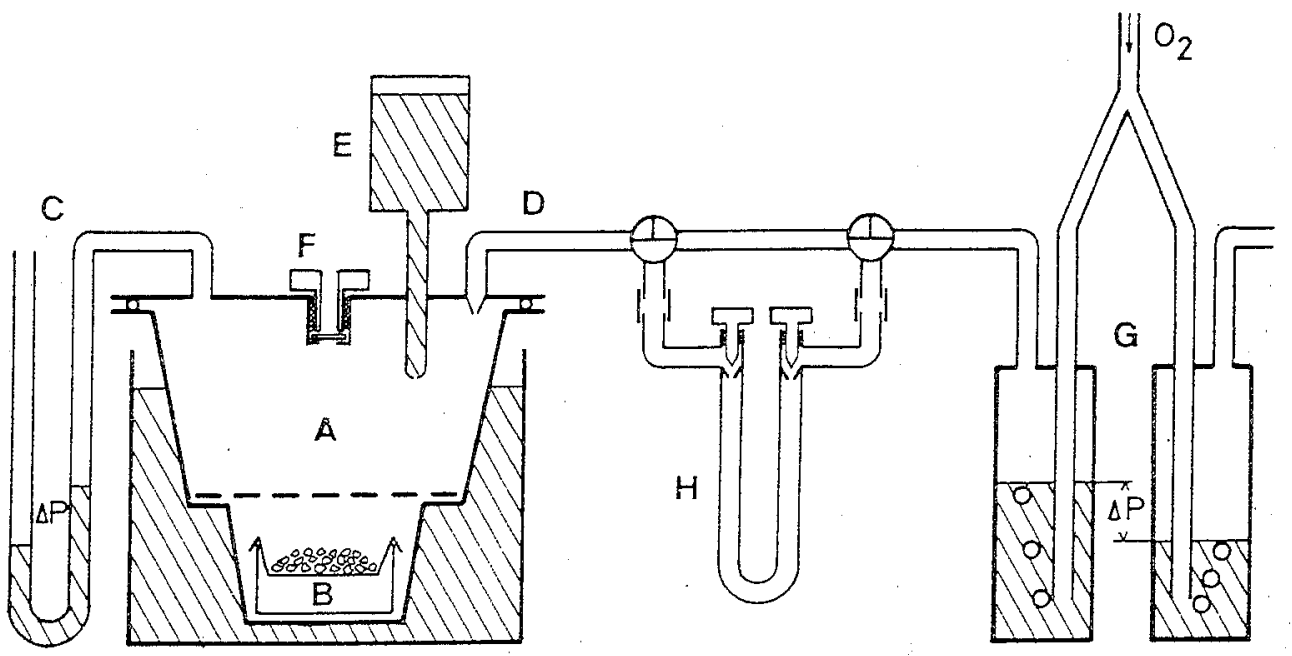

Fig. 1. Schematic view of the closed inhalation system. See the text for a description of components and operation

The lid is a custom-made aluminium disk clamped to the desiccator and sealing the chamber with an 0-ring. Metal tubes, pressed into three holes of the lid, allow a connection to a simple $U$-type manometer $(C)$, the oxygen supply (D) and a water bottle or a thermometer $(E)$. A septum holder $(F)$ is glued into a central hole of the lid and allows a periodical sampling of the atmosphere with a glass syringe and a $25 \mathrm{G}^{5} / 8$ needle for the assay of the concentration of the chemical by gas chromatography.

The oxygen is supplied from a needle valve to a split feeding two gas washing bottles (G) in parallel. The one on the right in Figure 1 is open to the outside and filled with less water than the one which is connected to the chamber. Due to this difference in the water levels the oxygen is bubbling to the outside unless the pressure in the chamber is reduced because of the adsorption of carbon dioxide by the soda lime. This constant reduced pressure in the chamber ensures that no chemical can be lost and that air tightness can always be checked by closing the oxygen supply and observing the rising underpressure in the chamber by means of the manometer (C).

\section{Application of the Chemical}

I) The chemical under investigation can be injected directly through the septum (F) on to a piece of filterpaper fixed underneath the porcelain disk.

II) If gases or radioactive material from break-seal ampoules are used, the chemical can be brought into the chamber in the oxygen supply stream by means of the bypass shown in the middle of Figure 1: The U-tube $H$, detached from the system, is connected on one side to a vacuum pump, on the other to the break-seal ampoule. The tube is immersed under vacuum into liquid nitrogen and the seal is broken up. The chemical is trapped in the U-tube, the valves are closed and the tube is connected to the inhalation system. The oxygen flow is switched to the by-pass, the valves are opened, and the chemical is blown with the oxygen stream into the chamber. 


\section{Operation}

Temperature Control. Experiments with a $300 \mathrm{~g}$ rat have shown that the heat produced by the animal rises the temperature inside the chamber by 5 to $7^{\circ} \mathrm{C}$. It is therefore advisable to immerse the desiccator into a water bath equipped with a tap water cooling coil and a thermostatic heater. A temperature setting of $15-17^{\circ} \mathrm{C}$ will then keep the animal at some $20-24^{\circ} \mathrm{C}$.

Humidity. Because of the urine collected on the bottom of the chamber and the transpiration of the animal the relative humidity soon reaches saturation. We did not introduce any drying agent into the chamber because of the inevitable adsorption of the chemical investigated. With a rather low temperature setting in our cooling bath we can at least keep the absolute humidity on a tolerable level.

Duration of Exposure. It is advisable to acclimatize the animal to the chamber before exposing it to the chemical. This is best done by daily sessions of increasing duration until the animal seems to be used to the new surroundings. We have been keeping $300 \mathrm{~g}$ rats in the chamber without access to food or water for a maximum of $26 \mathrm{~h}$.

\section{References}

Gage, J.C.: In: Methods in Toxicology, p. 258. (G.E. Paget, ed.), Oxford and Edinburgh: Blackwell Scientific Publications, 1970 